\title{
Using the electron localization function to correct for confinement physics in semi-local density functional theory
}

\author{
Feng Hao, Rickard Armiento and Ann E. Mattsson
}

\section{Linköping University Post Print}

\section{Tweet}

N.B.: When citing this work, cite the original article.

Original Publication:

Feng Hao, Rickard Armiento and Ann E. Mattsson, Using the electron localization function to correct for confinement physics in semi-local density functional theory, 2014, The Journal of Chemical Physics, (140), 18, 18A536.

http://dx.doi.org/10.1063/1.4871738

Copyright: (C) 2014 AIP Publishing LLC

http://scitation.aip.org/content/aip

Postprint available at: Linköping University Electronic Press

http://urn.kb.se/resolve?urn=urn:nbn:se:liu:diva-106608 


\section{A|P|l $\begin{aligned} & \text { The Journal of } \\ & \text { Chemical Physics }\end{aligned}$}

\section{Using the electron localization function to correct for confinement physics in semi- local density functional theory}

Feng Hao, Rickard Armiento, and Ann E. Mattsson

Citation: The Journal of Chemical Physics 140, 18 A536 (2014); doi: 10.1063/1.4871738

View online: http://dx.doi.org/10.1063/1.4871738

View Table of Contents: http://scitation.aip.org/content/aip/journal/jcp/140/18?ver=pdfcov

Published by the AIP Publishing

\section{Articles you may be interested in}

mBEEF: An accurate semi-local Bayesian error estimation density functional

J. Chem. Phys. 140, 144107 (2014); 10.1063/1.4870397

Fully nonlocal kinetic energy density functionals: A proposal and a general assessment for atomic systems

J. Chem. Phys. 129, 074103 (2008); 10.1063/1.2968612

Electron confinement: Models of kinetic and exchange energy functionals

AIP Conf. Proc. 577, 70 (2001); 10.1063/1.1390179

Jacob's ladder of density functional approximations for the exchange-correlation energy

AIP Conf. Proc. 577, 1 (2001); 10.1063/1.1390175

Density matrices for itinerant and localized electrons with and without external fields

J. Math. Phys. 38, 2037 (1997); 10.1063/1.532198

\section{A|P| Journal of}

Journal of Applied Physics is pleased to announce André Anders as its new Editor-in-Chief 


\title{
Using the electron localization function to correct for confinement physics in semi-local density functional theory
}

\author{
Feng Hao, ${ }^{1, a)}$ Rickard Armiento, ${ }^{2}$ and Ann E. Mattsson ${ }^{1, b)}$ \\ ${ }^{1}$ Multi-Scale Science MS 1322, Sandia National Laboratories, Albuquerque, New Mexico 87185-1322, USA \\ ${ }^{2}$ Department of Physics, Chemistry and Biology (IFM), Linköping University, SE-58183 Linköping, Sweden
}

(Received 15 November 2013; accepted 7 April 2014; published online 24 April 2014)

\begin{abstract}
We have previously proposed that further improved functionals for density functional theory can be constructed based on the Armiento-Mattsson subsystem functional scheme if, in addition to the uniform electron gas and surface models used in the Armiento-Mattsson 2005 functional, a model for the strongly confined electron gas is also added. However, of central importance for this scheme is an index that identifies regions in space where the correction provided by the confined electron gas should be applied. The electron localization function (ELF) is a well-known indicator of strongly localized electrons. We use a model of a confined electron gas based on the harmonic oscillator to show that regions with high ELF directly coincide with regions where common exchange energy functionals have large errors. This suggests that the harmonic oscillator model together with an index based on the ELF provides the crucial ingredients for future improved semi-local functionals. For a practical illustration of how the proposed scheme is intended to work for a physical system we discuss monoclinic cupric oxide, $\mathrm{CuO}$. A thorough discussion of this system leads us to promote the cell geometry of $\mathrm{CuO}$ as a useful benchmark for future semi-local functionals. Very high ELF values are found in a shell around the $\mathrm{O}$ ions, and take its maximum value along the $\mathrm{Cu}-\mathrm{O}$ directions. An estimate of the exchange functional error from the effect of electron confinement in these regions suggests a magnitude and sign that could account for the error in cell geometry. ( 2014 AIP Publishing LLC. [http://dx.doi.org/10.1063/1.4871738]
\end{abstract}

\section{INTRODUCTION}

Kohn-Sham (KS) Density functional theory (DFT) $)^{1,2}$ has proven very successful in describing the physics of atoms, molecules, and solids for a wide range of applications across disciplines. Of central importance for KS DFT is the description of the exchange-correlation (xc) energy. Prior work by the present authors and others has with great success identified and addressed the "electronic surface error" in many semi-local xc functionals. The solution has been to identify regions in space where electronic surface physics is present and map these regions to a relevant reference model from which the exchange-correlation energy is taken. This scheme is intended as a natural extension of the local-density approximation (LDA), in that here an additional idealized surface model system is introduced alongside the uniform electron gas. A post-correction scheme based on this idea has been successfully applied for correcting errors for the surface energies in metal/metal-oxide adhesion ${ }^{4}$ and the vacancy formation energies in metals. ${ }^{5,6}$

The correction was subsequently generalized into the self-consistent Armiento-Mattsson 2005 functional (AM05), ${ }^{7,8}$ a functional on generalized gradient approximation (GGA)-form. ${ }^{9}$ The AM05 functional uses an index based on the density gradient to interpolate between bulk and surface-like regions. In bulk-type regions the LDA is

\footnotetext{
a)Electronic mail: hfeng413@gmail.com

${ }^{b)}$ Electronic mail: aematts@ sandia.gov
}

used, and in the surface region each point was mapped to a corresponding point in the Kohn-Mattsson edge electron gas model ${ }^{10}$ to which a suitable correlation was composed using data for jellium surfaces. ${ }^{11}$ AM05 exhibits an excellent structural description of solids ${ }^{12-14}$ and, unlike the GGAs preceding it, is in near perfect systematic improvement of LDA. It has also been shown that while not performing as well as more specialized chemistry functionals, AM05 performs approximately as well as other semi-local functionals on a selection of chemical reaction energies. ${ }^{15}$

It has previously been proposed by us $^{16-18}$ that the construction idea of the Armiento-Mattsson functional can be generalized to an arbitrary number of model systems and, in this way, describes a practical and semi-systematical way towards functionals with higher accuracy, for both solid state systems and systems containing free atoms and molecules. We want to emphasize that our ultimate goal is to construct a semi-local functional that gives high accuracy results both for traditional chemistry systems like individual atoms and molecules and traditional physics systems such as solids and liquids. This would provide us with a much needed functional for treating mixed systems accurately and computationally efficiently. We have named this the subsystem functional scheme. ${ }^{17}$ Specifically, the failure of common semi-local functionals to handle confined electrons ${ }^{19-23}$ should be of primary concern for a next-generation semi-local functional. In a previous work we have therefore more closely investigated the error made by semi-local functionals for a confined electron gas. ${ }^{18}$ In the present paper we extend this 
investigation to suggest a practical way to identify regions in space where a correction for the error due to electron confinement is needed by using the electron localization function (ELF). We also discuss how one can map a point in a real system onto the confined electron gas model, and how, in principle, such a mapping is intended to work for a real physical system $(\mathrm{CuO})$.

In this context we briefly remark on the extensive library of beyond-DFT methods that successfully address errors that are arguably related to confined electrons in that they often resolve the tendency of currently used semi-local DFT functionals to give incorrectly over-delocalized states. In common use are, e.g., self-interaction correction, ${ }^{24-26}$ exact exchange and hybrid DFT methods, ${ }^{27-29}$ many-body Green's function $(\mathrm{GW}),{ }^{30}$ model potentials, ${ }^{31-36}$ and the addition of an effective Hubbard-like term to the Hamiltonian $(\mathrm{DFT}+U) .{ }^{37}$ However, we strongly emphasis that unlike those methods, the general and semi-systematic approach towards improved xc functionals discussed in the present work is completely within KS DFT, and the error we aim to address is clearly visible in the energy density as pertaining to regions in space where electrons are in presence of a confining potential. No immediate relation between this correction and that of the beyond-DFT methods is clear to us. None of the beyond-DFT methods are easily interpreted as directly modifying the xc energy density, and hence, it is questionably to discuss their corrections to the energy as done in a specific region in space (with the possible exception of model potentials). In particular, in DFT $+U$, the Hamiltonian is extended by a term involving the overlap projection of the KS orbitals onto atomic orbitals of some ions in the system. There is no reason to construe the correction achieved by this process as necessarily relating to a change in the xc energy density in the region close to those species. Nevertheless, we believe there is some relation between the errors we address in the present work from regions of confined electrons and the errors addressed by the beyond-DFT methods.

The errors in DFT approximations are usually assessed by calculating geometry properties, such as equilibrium lattice constants and bulk moduli, for a large set of materials spanning a broad range of different physical situations (i.e., bond types and arrangements). However, in many strongly correlated materials a broad range of different physical situations coexists near equilibrium. While the most usual sign of this coexistence is many different phases, there are a few materials where this coexistence and errors in functionals give an unusually clear signature in severely erroneous geometries.

This is the main motivation for discussing the properties of transition metal oxides, and, specifically, $\mathrm{CuO}$, in the later part of this paper. These systems have peculiar magnetic and structural properties that are, in general, not well described by semi-local DFT. These issues have been attributed to a JahnTeller-like distortion caused by a localized unpaired $d$ electron in $\mathrm{Cu}^{2+} \mathrm{O}^{2-} \cdot{ }^{38}$ Both the issues with magnetic states, and the cell geometry, have been successfully addressed by beyondDFT methods. ${ }^{39-41}$ This suggests that the cell geometry of the $\mathrm{CuO}$ system is a straightforward test case for exploring the errors made by semi-local functionals in regions of elec- tron confinement, and how these errors relate to beyond-DFT methods.

Hence, the paper presents a discussion of the influence of regions with high ELF values and confined electrons, that spans both the harmonic oscillator model, which can be thought of as a very idealized model of the electron physics in a small molecule, and solid state systems. We emphasis, again, that functional development of universal functionals applicable to problems across traditional chemistry and physics systems should consider both types of systems.

The rest of the paper is organized as follows. In Sec. II we discuss the ELF and its relevant properties in the context of confined electrons. In Sec. III we apply the ELF to a basic model of a confined electron gas based on the harmonic oscillator. We find that very high values of ELF coincide with regions in space where commonly used semi-local functionals deviate significantly from the exact xc energy density. In Sec. IV we discuss how, in principle, semi-local information can be used to map points in a general system to our confined electron gas model to estimate the error in the xc energy. In Sec. V we discuss how the interesting case of the cell geometry of cupric oxide, $\mathrm{CuO}$, makes for a good benchmark for future semi-local functionals. We then use the case of $\mathrm{CuO}$ to illustrate how the ideas presented in this paper apply to a real physical system. In Sec. VI, we summarize our results and give a few concluding remarks.

\section{THE ELECTRON LOCALIZATION FUNCTION}

As explained in the Introduction, in this work the ELF will take a major role in identifying regions in space where a correction based on the confined electron gas is required. The ELF is an index constructed by Becke and Edgecombe ${ }^{42}$ as a measure of the probability for finding an electron in the neighborhood of another electron with the same spin, thus providing a quantitative description of the Pauli exclusion principle. The ELF is expressed as

$$
\mathrm{ELF}=\frac{1}{1+\left(D / D_{h}\right)^{2}},
$$

where

$$
D=\tau_{\sigma}-\frac{1}{4} \frac{\left|\nabla n_{\sigma}\right|^{2}}{n_{\sigma}}, \quad D_{h}=\frac{3}{5}\left(6 \pi^{2}\right)^{2 / 3} n_{\sigma}^{5 / 3},
$$

with $n_{\sigma}(\mathbf{r})$ the density of electrons with spin $\sigma$, and $\tau_{\sigma}$ the kinetic energy density of electrons with spin $\sigma$,

$$
n_{\sigma}=\sum_{i}\left|\phi_{\sigma, i}\right|^{2}, \quad \tau_{\sigma}=\sum_{i}\left|\nabla \phi_{\sigma, i}\right|^{2},
$$

given as sums over all occupied Kohn-Sham orbitals with spin $\sigma, \phi_{\sigma, i}$. The formulas for a non-spin resolved system are readily obtained by setting $n_{\sigma}=n / 2$ and $\tau_{\sigma}=\tau / 2$, the spin-resolved values. The second term in $D$ is the boson kinetic energy density for a system with density $n_{\sigma}$ and it is also the minimum kinetic energy density that a fermion system can have. In the chemistry community this term is called the von-Weisäcker term and is usually interpreted as a single electron kinetic energy. $D_{h}$ is the value of $D$ for the uniform electron gas. The definition of the kinetic energy density is 
not unique. ${ }^{43}$ One can relate different definitions giving the same total kinetic energy by, e.g., a partial integration. However, since $D$ is the difference between two terms, the full and the von-Weisäcker kinetic energy densities, it is independent of the definition chosen as long as the same is used for both terms. By using the same definition also for $D_{h}$, the ELF becomes not only independent of definition of the kinetic energy density but also of units. ELF is a uniquely defined dimensionless quantity. In the explicit formulation above we have used the positive definite definition of kinetic energy density and Hartree units. $D$ is sometimes called the Pauli kinetic energy density, ${ }^{44}$ and it tends toward 0 when electrons are localized. The ELF only takes values in the range between 0 and 1 , where ELF $=1$ corresponds to the perfect localization and $\mathrm{ELF}=1 / 2$ is for the uniform electron gas. While the well known ELF index has been found very useful in describing and visualizing the atomic shells and chemical bonds in both molecules and solids, ${ }^{42,44-46}$ it is still useful for us to examine this object more closely to find an understanding applicable for both atoms and molecules and condensed matter systems. We base our interpretation on the fermion picture described in Ref. 45.

The ELF is an index applicable for all fermions, which is of interest in our case since the KS particles are fermions (though, not necessarily equivalent to electrons.) The ELF is measuring the fermionic nature of a system in different parts of space. The function $D$ is the extra amount of kinetic energy density of a fermionic system as compared to a bosonic system with the same density. Due to the Pauli exclusion principle, fermions need to all have different quantum numbers, i.e., be in different states, while any number of bosons can all be in the same state, and at zero temperature they form a bose condensate. Since fermions of different spin already fulfill the Pauli exclusion principle, the ELF is only a useful measure for same spin fermions, as is indicated in Eqs. (2) and (3). The implementation of ELF in the VIENNA $a b$ initio SIMULATION PACKAGE (VASP) versions we used (see below) is based on the non spin-resolved formulas in Ref. 45. For spin resolved densities this implementation does not give the same as the formulas from Ref. 42 (Eqs. (1)-(3) above) that we use in this work and the ELF values in the spin resolved calculations we performed had to be transformed by a post correction.

It is useful to consider the division in energy between potential and kinetic energy in a simple independent particle model, at zero temperature. Let us first consider the uniform electron/fermion gas. In such a gas all fermions have the same potential energy and, thus, in order to occupy different states, the fermions need to all have different kinetic energy. In a bose gas with a similar uniform density, all the bosons have the same, zero, kinetic energy. On the other hand, in a non-uniform potential, e.g., a columbic one, the fermions will all have different kinetic energies because quantization gives them orbitals that predominantly have different potential energies. A bose gas with the same density would have to occupy the same quantized orbitals as the fermions, thus the fermion and boson systems with the same density will have the same kinetic energy density. The fermions in their quantized orbitals do not need to adjust their kinetic energy in order to avoid each other. Their kinetic energy den- sity becomes bosonic, the ELF is close to 1 and we have localized states. Far outside a fermion system, in the classically forbidden region, we can only encounter the fermions with the highest kinetic energy, and the ELF goes to zero in most cases (see Ref. 42 for a discussion about exceptions). The ELF thus gives a clear division of space in a fermion system into regions where different types of physics dominate.

Before we give numerical results for the performance of functionals in different regions identified by values of ELF, we can already make some general observations: (i) high ELF regions need a functional that can treat localized electrons, (ii) intermediate ELF regions are likely to be accurately described by functionals based on the uniform electron gas, and (iii) in low ELF regions we need a functional that can treat the pure quantum effects present in the classically forbidden region, i.e., outside electronic surfaces. Hence, we expect LDA to work well in regions of type (ii), and the AM05 to work well in regions of type (ii) and (iii). However, there is no such a priori reason to expect either of these functionals to accurately describe the xc energy density in regions of type (i).

\section{THE ELF AS AN INDEX OF CONFINEMENT PHYSICS}

The general failure of conventional semi-local functionals for quasi-two-dimensional systems has been extensively discussed in the literature. ${ }^{19-23}$ In this section we discuss how the ELF directly identifies local regions in space responsible for this failure.

We take as model of a confined electron gas the quasione-dimensional harmonic oscillator (HO), in which electrons are constrained by a parabolic potential in one dimension (denoted by $z$ ) and are free to move in the other two, i.e., $v_{\text {eff }}(\mathbf{r})=\frac{\omega}{2} z^{2}$, where $\omega$ represents the potential strength. A recent work by us ${ }^{18}$ shows that all HO models can be classified by an occupation index $\alpha$, which indicates how many quantum levels in the $z$ dimension electrons can occupy. This index also gives a good quantification for the level of confinement: large values of $\alpha$ suggest a wide opening of the potential and thus a weak influence of confinement, whereas small values of $\alpha$ means that only a few quantum levels in $z$ are occupied, implying a stronger confinement. It is on these grounds we suggest the $\mathrm{HO}$ model as a useful reference system to quantify the confinement errors and correct for them in calculations of real systems.

In Figs. 1(a)-1(d) a series of HO models are shown with increasing $\alpha$. The $x$-axis is the dimensionless $z$-coordinate, $\bar{z}$, obtained by dividing $z$ with the characteristic length in the HO, $l=\sqrt{1 / \omega}$ (see Ref. 18 for detailed definitions of $z, l$, and $\bar{z}$ ). The corresponding local values of ELF are plotted in the upper half of each subfigure and we see that the value of ELF towards the center of the potential is closely correlated to the level of confinement in HO systems. In the lower part of each subfigure, the exact exchange energy per particle (given in dimensionless form, $l \epsilon_{\mathrm{x}}$ ) is shown together with the approximations by LDA and AM05. 
(a)

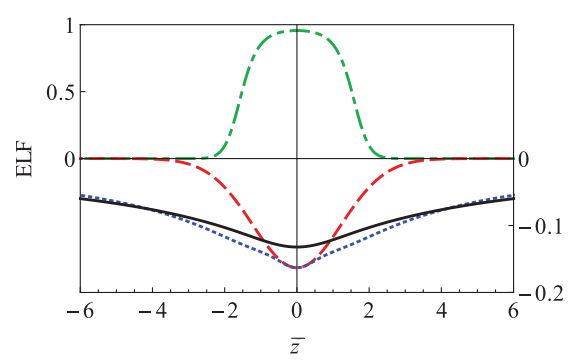

(c)

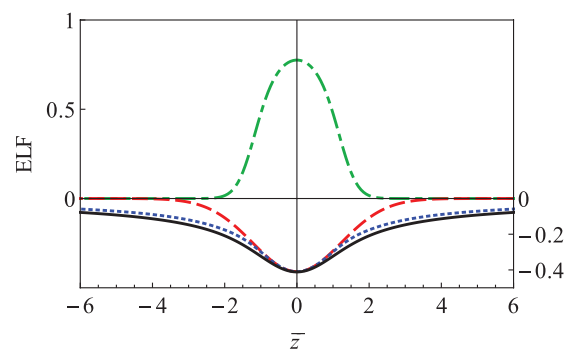

(b)

$\alpha=0.23$

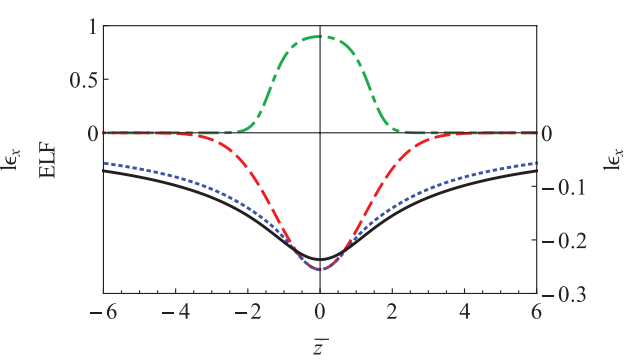

(d)

$\alpha=5.40$

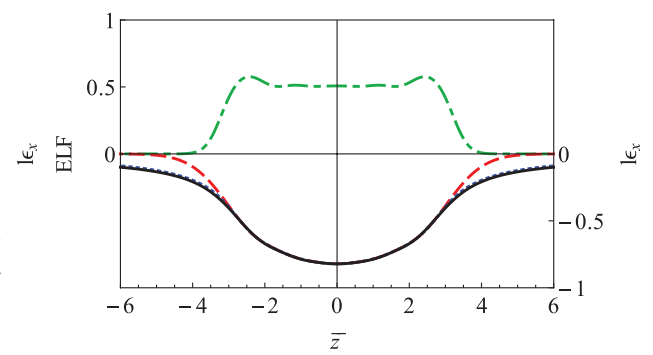

FIG. 1. [(a)-(d)] The ELF and the dimensionless exchange energy per particle $l \epsilon_{\mathrm{x}}$ for HO systems with different $\alpha$ as a function of $\bar{z}$. Dashed-dotted (green) lines show the ELF. The exact $l \epsilon_{\mathrm{x}}$ and approximations by LDA and AM05 are represented by solid (black), dashed (red), and dotted (blue) lines, respectively.

For the strongly confined systems with small $\alpha$, i.e., panels (a) and (b), both LDA and AM05 produce $l \epsilon_{\mathrm{x}}$ that is more negative than the exact one at the center of the potential well $(|\bar{z}| \approx 0)$. We interpret these differences as confinement errors in the functionals. The errors increase with decreasing $\alpha$. Figure 1 suggests a close correlation between large ELF values, i.e., $\gtrsim 0.8$, and a significant confinement error. This matches well the conclusion from our discussion of ELF in Sec. II. The physics that the ELF was designed to identify is not well described by the uniform electron gas, nor by the edge electron gas.

The first three subfigures, Figs. 1(a)-1(c), all have $\alpha<1$ and are thus so highly confined that only the lowest energy level from the $z$-dimension (HO) part of the potential is filled. For these systems, the maximal ELF is at $\bar{z}=0$ and is 0.96 , 0.90 , and 0.78 corresponding to $\alpha$ values of $0.06,0.23$, and 0.95 , respectively. In the third subfigure for $\alpha=0.95$, the system is close to accessing the second energy level in the $z$ dimension, and the confinement errors are now difficult to distinguish. As $\alpha$ further increases, e.g., in Fig. 1(d) we show $\alpha=5.40$, the $\mathrm{HO}$ gas behaves like a uniform electron gas in the region around the center of the harmonic potential, with the ELF taking its uniform electron gas value of $1 / 2$ and the value of LDA is indistinguishable from the exact result. We also note that the maximum ELF for the $\alpha=1$ system (not shown) is 0.77 , which we will use below as a "cutoff value" of regions where confinement physics is relevant.

Some past works have suggested that the density Laplacian, $\nabla^{2} n$, could also be used to identify errors for the xc energy in the strongly inhomogeneous electron gas. ${ }^{47-50}$ Nekovee and co-workers ${ }^{47,48}$ used the Variational Quantum Monte Carlo (VMC) method to investigate the xc energy density $e_{\mathrm{xc}}(\mathbf{r})=n(\mathbf{r}) \epsilon_{\mathrm{xc}}(\mathbf{r})$ of strongly inhomogeneous electron gases subject to sinusoidal external potentials. Subtracting the xc energy density obtained by VMC from the one obtained by using LDA, $e_{\mathrm{xc}}^{\mathrm{LDA}}-e_{\mathrm{xc}}^{\mathrm{VMC}}$, negative errors were shown to arise in the center of the quantum wells, which is consistent with our results for $\mathrm{HO}$ gas. They also found that the magnitude and the sign of the errors are correlated to the Laplacian of the electron density. A negative density Laplacian is found in the central region of the quantum wells. In the HO systems, a similarly negative Laplacian is also found with the appearance of large confinement errors (not shown). Cancio and $\mathrm{Chou}^{50}$ used the Laplacian as a correction in bulk Si. However, given the substantial amount of prior work that backs up the interpretation of the ELF as identifying regions in space with localized electrons, it appears to us to be a more straightforward and useful option, in particular in combination with the interpretation of the ELF as an indicator of the fermionic character of different parts of the system.

\section{MAPPING INTO THE MODEL SYSTEM}

It is not clear to us, at this point, how general the quantitative difference between the functionals and the exact result found in the harmonic oscillator confined electron gas model is. It is possible that other features in the potential than the characteristic length-scale of the most strict confinement affect the magnitude of the error we aim to correct. Nevertheless, in this section we show how a point in a general system can be mapped into our model system based only on semilocal information. Should we in future work find that the HO model is too limited to give a sufficiently general quantification of the error, this mapping will need to be extended to a more complex model system.

Our goal in the following is to map a point in a general system onto a specific point in our model system, i.e., a specific value of $\alpha$ and $\bar{z}$. We take the point in the real system to 
have the following two dimensionless semi-local quantities:

$$
\begin{gathered}
s=\frac{|\nabla n|}{2\left(3 \pi^{2}\right)^{1 / 3} n^{4 / 3}}, \\
t=\frac{\tau}{(3 / 10)\left(3 \pi^{2}\right)^{2 / 3} n^{5 / 3}} .
\end{gathered}
$$

From Fig. 1 we find that the magnitude of the confinement error is only relevant for $\alpha<1$, and hence we restrict the map to only apply for such values of $\alpha$. For this case, the density and kinetic energy take these simple forms

$$
\begin{aligned}
& {\left[l^{3} n(\alpha, \bar{z})\right]=\frac{1}{\pi^{3 / 2}} e^{-\bar{z}^{2}} \alpha,} \\
& {\left[l^{5} \tau(\alpha, \bar{z})\right]=\frac{1}{2 \pi^{3 / 2}} e^{-\bar{z}^{2}}\left(\alpha^{2}+\alpha \bar{z}^{2}\right),}
\end{aligned}
$$

which can readily be inverted:

$$
\begin{gathered}
\bar{z}^{2}=\frac{1}{2} W\left[18 \pi\left(\frac{3}{5} t-s^{2}\right)^{2} s^{2}\right], \\
\alpha=\bar{z}^{2}\left(\frac{3}{5} \frac{t}{s^{2}}-1\right) .
\end{gathered}
$$

These expressions are defined for any given pair of $s$ and $t$, and thus the general map is complete. However, it is only relevant to use it for regions in space with ELF values comparable to those we find in a confined electron gas model with $\alpha<1$, i.e., an $\mathrm{ELF} \gtrsim 0.77$.

\section{CUPRIC OXIDE}

In this section we demonstrate how an ELF-based index and the mapping into the confined electron gas model is intended to work in practice. For a relevant and illustrative example we will use monoclinic cupric oxide, $\mathrm{CuO}$. In the following we first discuss the interesting properties of $\mathrm{CuO}$, and then try a provisional estimate of the confinement error for this system.

Transition-metal oxides (TMMOs) are in general difficult to treat with semi-local DFT methods. It has been found that LDA and the common GGAs give incorrect ground state properties, such as incorrect magnetic moments and an imprecise representation of the band structure. ${ }^{51-54}$ This is commonly attributed to the incorrect description of the localized $3 d$ electrons, and have been successfully addressed using the beyond-DFT methods we mentioned in the Introduction. For example, Engel and Schmid have shown that exact exchange and LDA correlation produce reasonable results for the magnetic moments and band gap calculations. ${ }^{40,41}$

As a more straightforward problem than the magnetic and band gap properties of TMMOs, we will focus on the equilibrium structure of $\mathrm{CuO}$. As opposed to the cubic rock-salt equilibrium structure of the TMMOs with lower atomic numbers, $\mathrm{CuO}$ exhibits a less symmetric monoclinic structure in equilibrium. This structure is shown in $2 \mathrm{D}$ and $3 \mathrm{D}$ in Figs. 2 and 3. Due to the ground state magnetic state, the primitive unit cell has $8 \mathrm{Cu}$ and $8 \mathrm{O}$ atoms shown in Fig. 2. The atoms are numbered consistently in both figures. The peculiar structure of $\mathrm{CuO}$ has been attributed to a Jahn-Teller-like

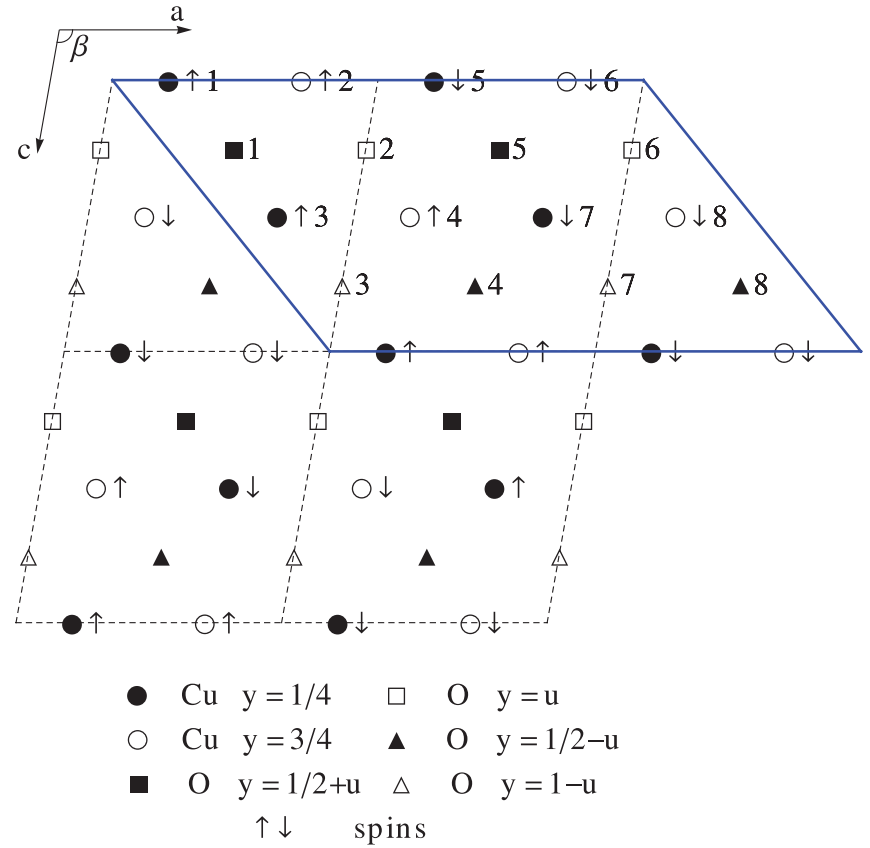

FIG. 2. The equilibrium structure of $\mathrm{CuO}$ in the $(\mathrm{a}, \mathrm{c})$ plane. The other principal axis, $\mathrm{b}$, is perpendicular to the plane. Black dashed lines encircled the nonmagnetic unit cell. The angle between axes a and $\mathrm{c}$ is $\beta$. The internal parameter $u$ characterizes the position of the oxygen atom plane in a unit cell. The solid (blue) lines encircle the supercell used for the DFT calculation, including $8 \mathrm{Cu}$ and $8 \mathrm{O}$ atoms, indicated by numbers. This supercell has accounted for the magnetic structure of the material. The sizes of the experimental cell parameters are: ${ }^{55} \mathrm{a}=4.6837 \AA, \mathrm{b}=3.4266 \AA$, $\mathrm{c}=5.1288 \AA ⿻$ $=99.54^{\circ}$, and $u=0.4184$

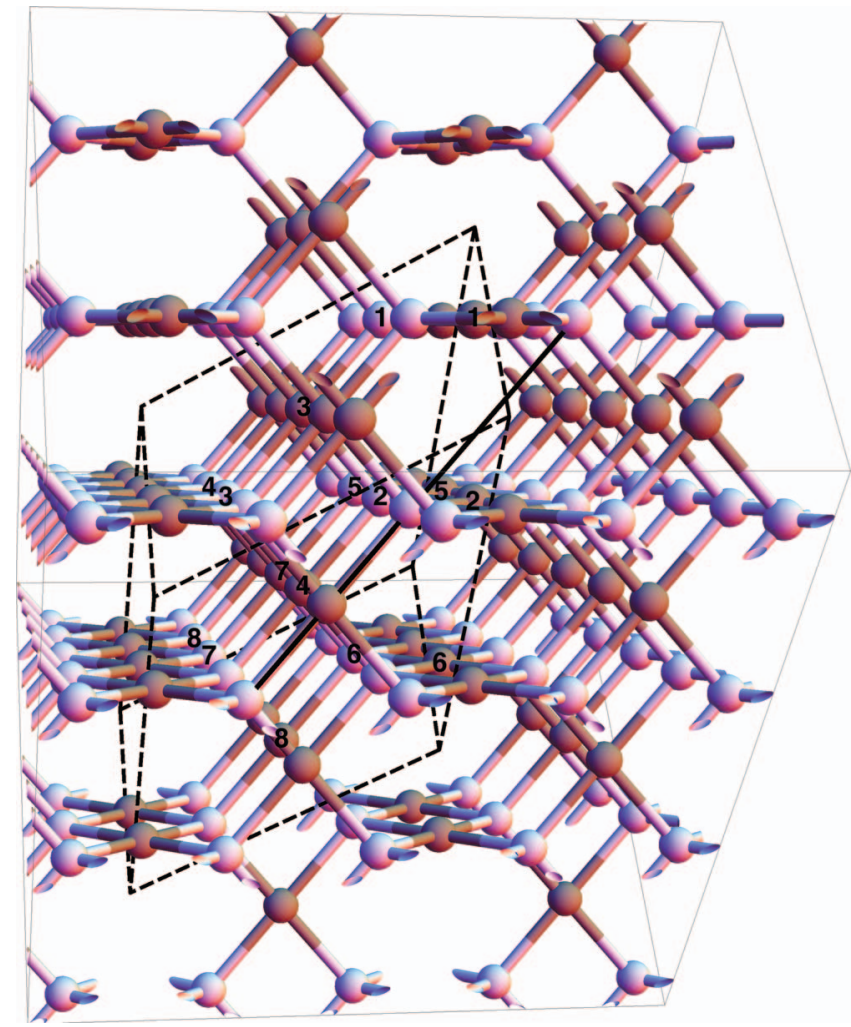

FIG. 3. 3D view of the $\mathrm{CuO}$ lattice structure that has experimental volume but with internal parameters $c / a=1.23, b / a=1.00, \beta=90^{\circ}, u=0.5$. The copper and oxygen atoms are shown with dark (brown) and light (purple) colors. Black dashed lines indicate the supercell used in the simulation with the same numbering of atoms in the solid line (blue) box shown in Fig. 2. 
distortion caused by the unpaired $d$ electron in $\mathrm{Cu}^{2+} \mathrm{O}^{2-}$, which is also related to the magnetic moments and band structure of $\mathrm{CuO}{ }^{38}$

The equilibrium structures calculated by LDA and common semi-local functionals are not the experimentally obtained tilted monoclinic structure, but instead a more symmetric rectangular structure with larger lattice constants than expected (note that this rectangular structure is different from the rock-salt structure, which has an even higher total energy). It appears likely that the source of this unusually large discrepancy in geometry found with semi-local methods is the same as the other difficulties to describe TMMOs. Wu, Zhang, and Tao indeed report a much improved $\mathrm{CuO}$ geometry when using DFT $+U .{ }^{39}$ This is unusual, since, in general, the representation of ground state geometry of crystals is often very accurate in semi-local DFT. Hence, the failure of the commonly used functionals to correctly describe the ground state of $\mathrm{CuO}$ provides an unusually straightforward test case for functional development that targets the issues present for TMMOs, which is not subject to any ambiguity with respect to, e.g., the interpretation of the KS orbitals.

In the following we will use the ELF to identify regions in $\mathrm{CuO}$ that are likely to have a large confinement error and then use the mapping suggested above to provide a rough estimate of the errors in the system. The result seems to indicate that the error discussed in this work is large enough to possibly account for the incorrect geometry of $\mathrm{CuO}$. However, for this discussion we first need to make an observation about the relation of the cell volume and the rest of the cell geometry in $\mathrm{CuO}$, which, as far as we are aware, have not been present in prior discussion of this system.

\section{A. Cell volume and cell geometry in CuO}

Using the VASP ${ }^{56-58}$ code we have calculated the cell shape of $\mathrm{CuO}$ with three different functionals: the LDA, the Perdew-Burke-Ernzerhof (PBE) functional, ${ }^{59}$ and the AM05 functional. ${ }^{7,8}$ See Appendix A for further calculational details. We have performed these calculations at various fixed volumes characterized by the ratio between the volume used $V$ and the experimental volume $V_{0}$. Since we prefer a measure with the same dimension as the lattice constants, we will consistently use the scale, $\left(V / V_{0}\right)^{1 / 3}$.

The calculated relaxed structure parameters for different cell volumes and functionals are presented in Fig. 4. For large supercells, all three functionals give similar high symmetry rectangular structures with the angle between a and $\mathrm{c}$ axes $\beta$ $=90^{\circ}$, and the internal parameter $u$ around 0.5 (see Fig. 2 for definition of the parameters). As the cell volume is decreased, all functionals transition into structures that are tilted with $\beta$ larger than $90^{\circ}$ and the $\mathrm{O}$ atoms are shifted compared to the fixed $\mathrm{Cu}$ atom lattice with a $u$ smaller than 0.5.

Figure 5 shows the total energy versus volume for the relaxed structures with the different functionals. The calculated energy minimums are respectively at scales: 1.01 (LDA), 1.02 (AM05), and 1.04 (PBE). Fig. 4 shows that all three functionals give a similar structure at the equilibrium minimum
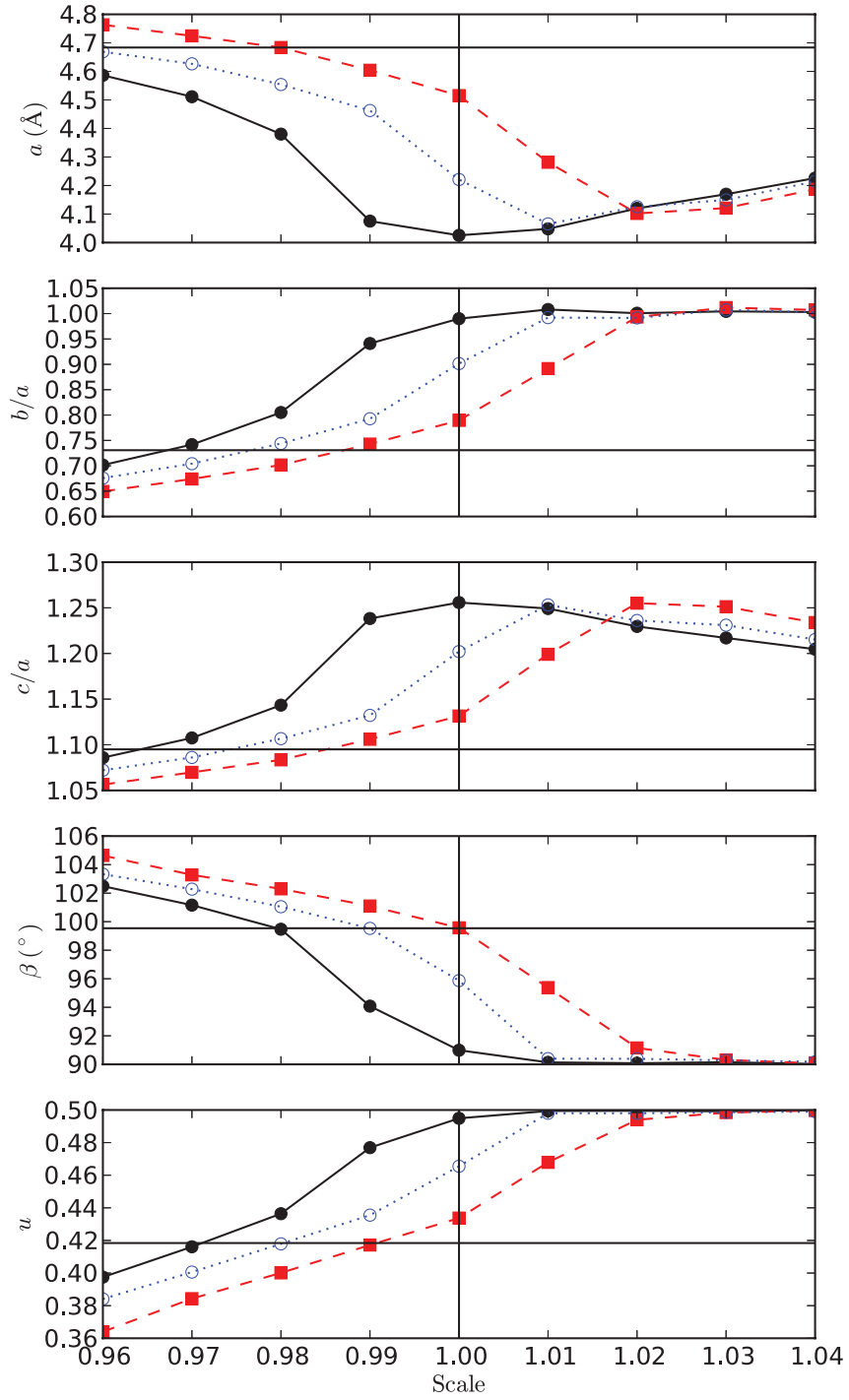

FIG. 4. Different structural parameters in $\mathrm{CuO}$ when all internal degrees of freedom are relaxed at fixed volume with the LDA (solid, black), AM05 (dotted, blue), and PBE (dashed, red) functionals. The equilibrium volumes are at scale 1.01 for LDA, 1.02 for AM05, and 1.04 for PBE. The experimental cell parameters are marked with straight black lines. For all functionals we find the structural parameters to greatly improve at compressed volumes, suggesting that if an xc correction moves the energy minimums towards smaller volumes, deviations from experimental values for the description of geometry would be more in line with the expectation for semi-local DFT.

energy volume, with $\beta \approx 90^{\circ}, u \approx 0.5, b / a \approx 1$, and $c / a$ $\approx 1.23$. Hence, none of these functionals reproduce the experimental monoclinic structure at their respective equilibrium. However, at compressed volume, all three functionals relax to a cell geometry very close to the experimental one. This suggests that if an xc energy correction move the energy minimums towards smaller volumes, errors of more usual magnitude for semi-local DFT would be achieved for all the cell parameters.

In Fig. 5, graphs are also present for energies for cells that have been held perfectly rectangular, i.e., $\beta=90^{\circ}, b / a=1, u$ $=0.5$, and where only $c / a$ is relaxed. At large scale/volume, the stable structures for fixed volume calculations are rectangular and the two lines coincide. For compressed cells, the cells with broken symmetry are lower in energy. These 


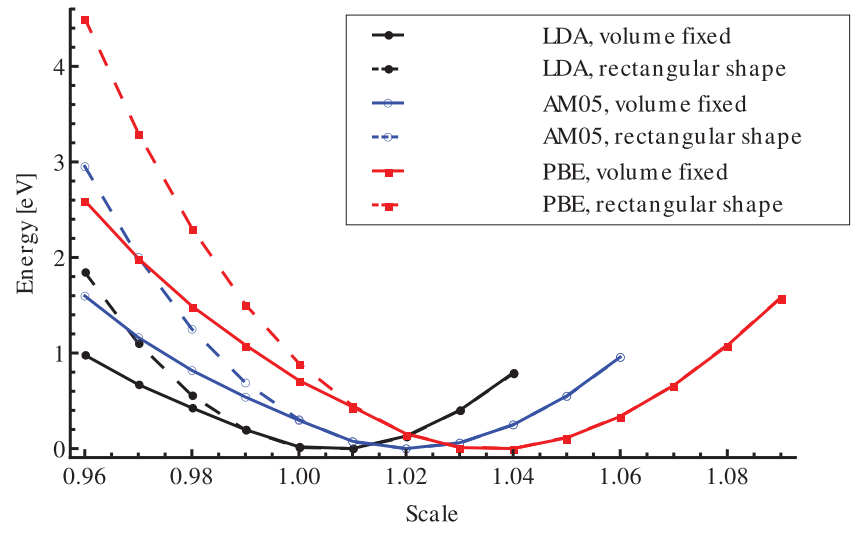

FIG. 5. Energy as function of the scale of the supercell with respect to the experimental structure. Solid lines are for calculations with volume fixed, and allow the relaxation of both the supercell shape and the atom positions. The dashed lines are for calculations with the further restriction that the structure has to be rectangular, i.e. $\beta=90^{\circ}, b / a=1.0$, and the internal parameter $u$ is 0.5 . Black, dark gray (blue), and light gray (red) are showing different functionals used for the calculations: LDA, AM05, and PBE. All graphs are aligned on their minimum point, taken to be 0 .

results suggest that the problem with the cell geometry in the semi-local functionals is not due to inability in describing the crucial Jahn-Teller-like distortion, since at the compressed volumes the structures with broken symmetry are lower in energy as they should. Rather, this further motivates that all functionals are missing some energy contribution that acts to decrease the cell volume.

It is well established that LDA systematically underestimates the cell volume, PBE overestimates it, and AM05 gives results that are in between them (see, e.g., Refs. 13 and 14). For $\mathrm{CuO}$, the equilibrium volumes appear in the same order, $\mathrm{PBE}>\mathrm{AM} 05>\mathrm{LDA}$. However, for $\mathrm{CuO}$ even the LDA equilibrium volume is larger than the experimental one. This is again consistent with our conjecture that all the functionals share a systematic error that leads to an overestimated equilibrium volume for $\mathrm{CuO}$.

In the following we will argue, but without absolute conviction, that this systematic error present in all functionals possibly is the confinement error discussed in Secs. I-IV of this paper. Our discussion is intended to demonstrate the use of the ELF as an index of confinement error and the use of the map of semi-local quantities to the confined electron gas.

\section{B. Estimating the confinement error in $\mathrm{CuO}$}

Figure 6 shows the ELF along the $\mathrm{Cu}-\mathrm{O}$ and $\mathrm{O}-\mathrm{O}$ directions and Fig. 7 shows 3D contour plots of ELF for both the experimental monoclinic and the restricted rectangular structure. The regions with high ELF $>0.77$ values are located in a shell around the $\mathrm{O}$ atom, and everywhere else the ELF is moderately low. We also note that the region of high ELF values is somewhat smaller in the rectangular structure than in the monoclinic structure.

A rough functional-dependent estimate of the confinement error can now be made by (1) identifying the regions in space where the ELF $>0.77$ (i.e., the previously mentioned

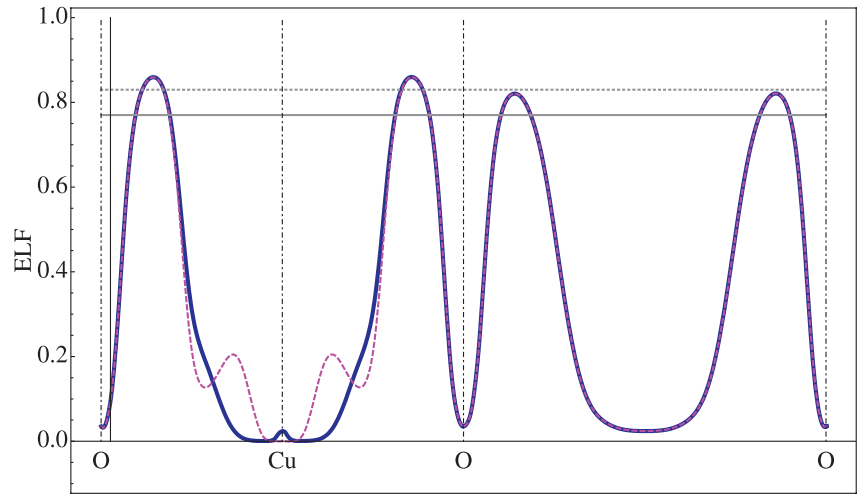

FIG. 6. The ELF plotted along a straight line passing through nearest neighbor $\mathrm{O}-\mathrm{Cu}-\mathrm{O}$ within a ribbon, and then passing the void to the oxygen in the next parallel ribbon (shown by solid line in Fig. 3). The two graphs are given using two different pseudopotentials (see Appendix A for details). The ELF is calculated on the lattice structure shown in Fig. 3 with the PBE functional. The horizontal lines show relevant values of ELF used in this work: 0.83 (dotted) and 0.77 (full). The dashed-dotted vertical lines show the positions of $\mathrm{Cu}$ and $\mathrm{O}$ ions. For comparison, the vertical full line shows the average displacement of a $1 s$ electron from the oxygen nucleus, derived from the hydrogen like atom (non-interacting electrons).

"cutoff" value that corresponds to $\alpha<1$ in the HO model), and (2) the use of the mapping procedure discussed in Sec. IV to find the error the same functional makes in the corresponding point in the confined electron gas model system. More details of the error estimate are given in Appendix B. The results are shown in Fig. 8. The crucial finding is that the errors grow near linearly with system volume. If this is indeed a feature that is correctly reproduced by the rather crude error estimate, it would be responsible for inflating the equilibrium cell volume of all the functionals.

To show this more clearly, Fig. 9 shows the total energy versus volume for the functionals corrected for the estimated errors. We find that the estimated error indeed is of a direction and magnitude sufficient to address the error in cell volume. Removing the estimated error also appears to bring AM05 and PBE quite close together.
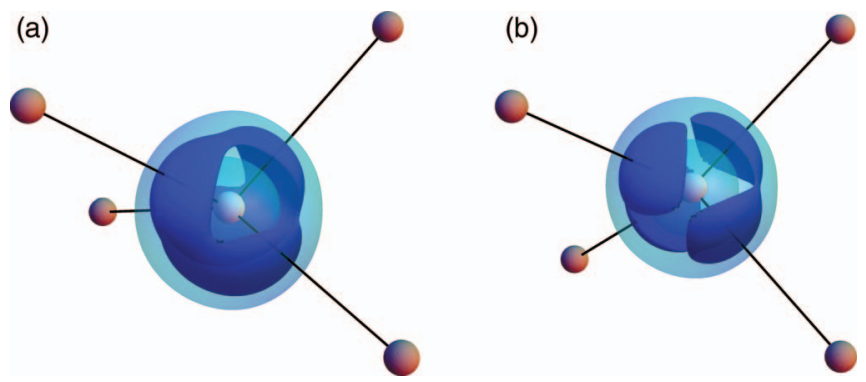

FIG. 7. Three-dimensional contour plot of ELF for $\mathrm{CuO}$ with approximately the experimental monoclinic (a) and rectangular structure (b). Both structures have volumes that are $0.97^{3}$ of the experimental structure. For the rectangular structure, the internal parameters have been restricted to be $c / a=1.23, b / a$ $=1.0, \beta=90^{\circ}$, and $u=0.5$. The LDA functional is employed to calculate the ELF values. The contour is surrounding spacial regions where the ELF value is larger than 0.83 (dark blue) and 0.77 (light blue). $4 \mathrm{Cu}$ atoms (dark; brown) connect to the one $\mathrm{O}$ atom (light; gray) in the center. The two $\mathrm{Cu}$ atoms on the right are in the same ribbon, and the left two are in another ribbon that crosses the former one (see Fig. 3). 


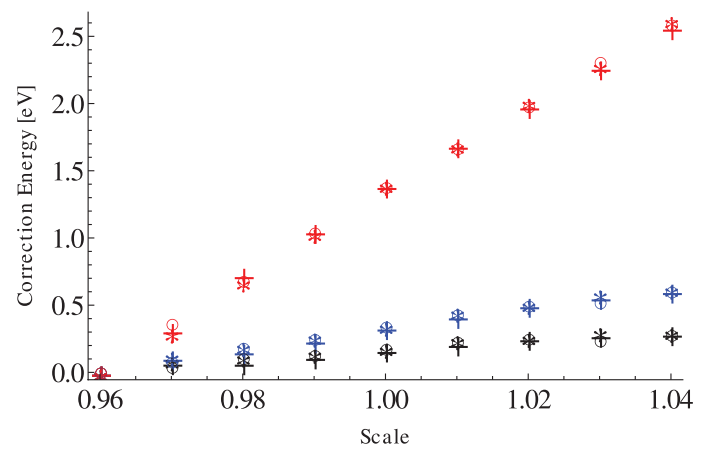

FIG. 8. Estimated confinement error in $\mathrm{CuO}$ for LDA (smallest values), AM05 (slightly larger values, blue), and PBE (largest values, red). Densities calculated with different functionals (“*” (LDA), "+" (AM05), and "o" $(\mathrm{PBE})$ ) give practically identical error estimates.

We emphasize that the analysis presented here of the confinement error in $\mathrm{CuO}$ is, at this point, quite exploratory. We intend with this discussion to promote $\mathrm{CuO}$ as a benchmark to probe physics that otherwise commonly requires a discussion of quantities that are more difficult to interpret in KS DFT (e.g., orbital features or band gaps). In any case, the error estimate serves as a useful example of how a more general correction scheme is intended to work. We find it encouraging that the magnitude and sign of our rather crude error estimate suggests that the effect is sufficient large and with the correct sign to correct the equilibrium geometry. However, we are cautious in stressing this point, since correcting total energies for high ELF value regions in the way done for $\mathrm{CuO}$ in other systems that are already well described by semilocal DFT can worsen cell geometries. For example, for $\mathrm{Si}$, "correcting" the cell volume using the error estimate of this work distorts the equilibrium volume by $\sim 0.05 \AA$. However, a good case can be made that this is simply an effect of the crudeness of the error estimate, and, especially, the lack selfconsistent treatment. A more appropriate assessment of the type of correction suggested in this work requires a general self-consistent treatment in line with the Armiento-Mattsson construction used for the AM05 functional.

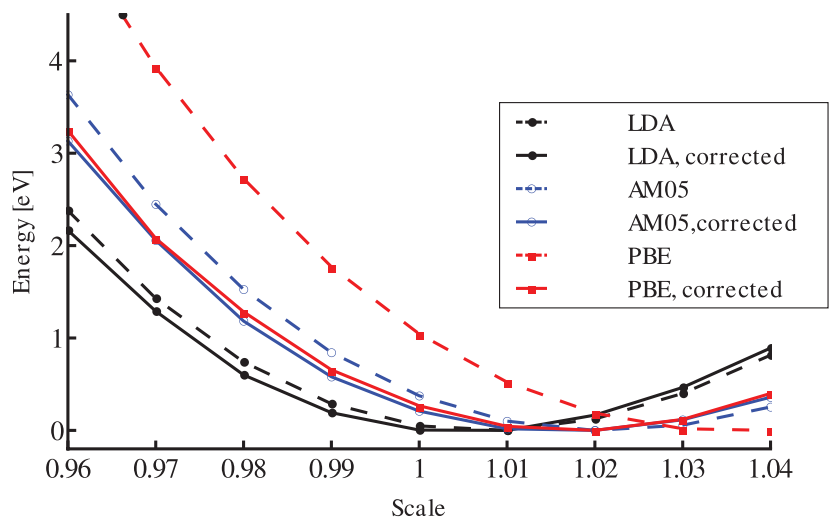

FIG. 9. The effect on the location of the energy minimum for the various functionals if the error estimate in Fig. 8 is used to correct rectangular shape values similar to those in Fig. 5, but for fixed $c / a=1.23$, (shown here as dashed lines.) All graphs are aligned on their minimum point, taken to be 0 .

\section{CONCLUSIONS}

In this paper we have discussed the use of the ELF as an index to identify regions in space with large errors in common semi-local functionals. Using a model system of an electron gas confined in one dimension, we have established a connection between very high values $(\gtrsim 0.77)$ of the ELF and large errors of such functionals. We have discussed how semi-local information in a point in a general system can be mapped into the confined electron gas to estimate the size of these errors. We then presented an in-depth analysis of the cell geometry error in $\mathrm{CuO}$ that promotes this system as a benchmark for future semi-local functionals aimed at errors that presently require beyond-DFT methods. The estimate of the error due to confined electrons was used to discuss the effect we expect an improved functional to have on $\mathrm{CuO}$. However, we stress again that the scheme used is not, at this point, intended as general recipe to be used indiscriminately for other systems. However, from the results presented here it now appears straightforward to extend the work in the direction of a general functional in the form of a meta-GGA that can be applied self-consistently (a meta-GGA is a GGA with an additional dependence on the kinetic energy density.) Such a functional would allow for the broader testing necessary to properly assess the accuracy of the proposed scheme for systems of interest to both the physics and chemistry communities.

\section{ACKNOWLEDGMENTS}

This work was supported by the Laboratory Directed Research and Development Program. Sandia National Laboratories is a multi-program laboratory operated by Sandia Corporation, a wholly owned subsidiary of Lockheed Martin company, for the U.S. Department of Energy National Nuclear Security Administration under Contract No. DE-AC0494AL85000. R.A. acknowledges support from the Swedish Research Council (VR), Grant No. 621-2011-4249 and the Linnaeus Environment at Linköping on Nanoscale Functional Materials (LiLi-NFM) funded by VR.

\section{APPENDIX A: CALCULATIONAL DETAILS}

Our DFT calculations are done using the VASP ${ }^{56-58}$ versions 5.1.49 and 5.2.5 with a plane wave basis set and the projector-augmented wave (PAW) method to describe the interactions between ions and electrons. In our calculations at fixed volume, we first performed two consecutive relaxations using gaussian smearing of the electron occupation with a width of $0.05 \mathrm{eV}$. Then a final static calculation is performed to obtain the exact energy using the tetrahedron method with Blöchl corrections. In all these calculations, to ensure the convergence of our results, a cutoff energy of $1050 \mathrm{eV}$ for the plane wave basis is used, and the Brillouin zone is sampled using a $6 \times 6 \times 6$ mesh of $k$ points in a Monkhorst-Pack scheme. ${ }^{60}$ The AM05 calculations are performed with PBE PAW potentials. ${ }^{13}$

Since much of this work relies on accurate calculation of the ELF using a pseduopotential code, we need to address whether the missing contribution to the ELF from core electrons could affect our results. In Fig. 6, we show the 
ELFs for two calculations with different pseudopotentials. The blue full curve is for calculation of ELF with 11 valence electrons for $\mathrm{Cu}$, and the magenta dashed line is obtained using $\mathrm{Cu}$ PAW potential that includes the extra $63 p$ core electrons, so having 17 valence electrons in total. The ELFs are shown along a straight line passing through the $\mathrm{O}-\mathrm{Cu}-\mathrm{O}$ atoms in one of the ribbons in the $\mathrm{CuO}$ structure and then through the void to connect to another oxygen atom in the next parallel ribbon. It is clear that the only difference by adding more $\mathrm{Cu}$ valence electrons is that the ELF is increased at the close vicinity of $\mathrm{Cu}$ atom, but still the ELF value is only around 0.2 , which will not add more regions that need confinement error correction. For the existing high ELF regions, the two calculations show the exact same results. As for the $\mathrm{O}$ atoms, we did not perform calculations with the two $1 s$ core electrons treated as valence, but we do show the average displacement of them from the oxygen nucleus as indicated by a vertical full line, which is far from the high ELF area. Obviously, even if the ELF contributed by them is large, it will contribute the same correction for all different fixed-volume calculations, and therefore will not make an impact on the volume-dependent energy correction.

\section{APPENDIX B: DETAILS OF THE ESTIMATE OF THE CONFINEMENT ERROR}

This Appendix gives the detailed formulas used to estimate the confinement error for $\mathrm{CuO}$.

(i) For every grid point in the calculation, find those where the ELF $>0.77$, the previously mentioned "cutoff" value that corresponds to $\alpha<1$ in the HO model.

(ii) For each of these grid points, find the corresponding $\mathrm{HO}$ model parameters, $\alpha$ and $\bar{z}$, according to the mapping procedure in Sec. IV.

(iii) Calculate the relative difference between the exchange part of the density functional approximation (DFA) that was used and the exact exchange calculated in the $\mathrm{HO}$ model, based on the $\alpha$ and $\bar{z}$ obtained in step (ii):

$$
\Delta \epsilon_{\mathrm{x}}=\left[l \epsilon_{\mathrm{x}}^{\mathrm{HO}}(\alpha, \bar{z})\right] /\left[l \epsilon_{\mathrm{x}}^{\mathrm{DFA}}(\alpha, \bar{z})\right]-1,
$$

where $l \epsilon_{\mathrm{x}}^{\mathrm{HO}}(\alpha, \bar{z})$ is the dimensionless exact exchange energy per particle of the HO gas, and can be numerically obtained via Eq. (16) in Ref. 18. The $l \epsilon_{\mathrm{x}}^{\mathrm{DFA}}(\alpha, \bar{z})$ is the approximation of $l \epsilon_{\mathrm{x}}^{\mathrm{HO}}(\alpha, \bar{z})$ by different functionals in the HO gas.

(iv) Estimate the total confinement error in the exchange energy as an integration over all the grid points where $\mathrm{ELF}>\mathrm{ELF}_{\mathrm{c}}$,

$$
E_{\mathrm{x}}^{\mathrm{err}}=\int_{\mathrm{ELF}>\mathrm{ELF}_{\mathrm{c}}} d \mathbf{r} n(\mathbf{r}) \epsilon_{\mathrm{x}}^{\mathrm{DFA}}(\mathbf{r}) \Delta \epsilon_{\mathrm{x}}(\mathbf{r}),
$$

where $\epsilon_{\mathrm{x}}^{\mathrm{DFA}}(\mathbf{r})$ is calculated based on the semi-local information in the real $(\mathrm{CuO})$ system.

\footnotetext{
${ }^{1}$ P. Hohenberg and W. Kohn, Phys. Rev. 136, B864 (1964).

${ }^{2}$ W. Kohn and L. J. Sham, Phys. Rev. 140, A1133 (1965).

${ }^{3}$ A. E. Mattsson and W. Kohn, J. Chem. Phys. 115, 3441 (2001).

${ }^{4}$ A. E. Mattsson and D. R. Jennison, Surf. Sci. Lett. 520, L611 (2002).

${ }^{5}$ K. Carling, G. Wahnström, T. R. Mattsson, A. E. Mattsson, N. Sandberg, and G. Grimvall, Phys. Rev. Lett. 85, 3862 (2000).
}

${ }^{6}$ T. R. Mattsson and A. E. Mattsson, Phys. Rev. B 66, 214110 (2002)

${ }^{7}$ R. Armiento and A. E. Mattsson, Phys. Rev. B 72, 085108 (2005).

${ }^{8}$ A. E. Mattsson and R. Armiento, Phys. Rev. B 79, 155101 (2009).

${ }^{9}$ J. P. Perdew and Y. Wang, Phys. Rev. B 33, 8800 (1986).

${ }^{10}$ W. Kohn and A. E. Mattsson, Phys. Rev. Lett. 81, 3487 (1998).

${ }^{11}$ Z. Yan, J. P. Perdew, and S. Kurth, Phys. Rev. B 61, 16430 (2000).

${ }^{12}$ A. E. Mattsson, R. Armiento, and T. R. Mattsson, Phys. Rev. Lett. 101, 239701 (2008).

${ }^{13}$ A. E. Mattsson, R. Armiento, J. Paier, G. Kresse, J. M. Wills, and T. R. Mattsson, J. Chem. Phys. 128, 084714 (2008).

${ }^{14}$ F. Tran, R. Laskowski, P. Blaha, and K. Schwarz, Phys. Rev. B 75, 115131 (2007).

${ }^{15}$ R. P. Muller, A. E. Mattsson, and C. L. Janssen, J. Comp. Chem. 31, 1860 (2010).

${ }^{16}$ A. E. Mattsson and R. Armiento, Int. J. Quantum Chem. 110, 2274 (2010).

${ }^{17}$ R. Armiento and A. E. Mattsson, Phys. Rev. B 66, 165117 (2002).

${ }^{18}$ F. Hao, R. Armiento, and A. E. Mattsson, Phys. Rev. B 82, 115103 (2010).

${ }^{19}$ P. Garcia-Gonzalez, Phys. Rev. B 62, 2321 (2000).

${ }^{20}$ P. Garcia-Gonzalez and R. W. Godby, Phys. Rev. Lett. 88, 056406 (2002).

${ }^{21}$ L. A. Constantin, J. P. Perdew, and J. M. Pitarke, Phys. Rev. Lett. 101, 016406 (2008).

${ }^{22}$ Y. H. Kim, I. H. Lee, S. Nagaraja, J. P. Leburton, R. Q. Hood, and R. M. Martin, Phys. Rev. B 61, 5202 (2000).

${ }^{23}$ L. Pollack and J. P. Perdew, J. Phys.: Condens. Matter 12, 1239 (2000).

${ }^{24}$ J. Perdew, Chem. Phys. Lett. 64, 127 (1979)

${ }^{25}$ J. P. Perdew and A. Zunger, Phys. Rev. B 23, 5048 (1981).

${ }^{26}$ R. O. Jones and O. Gunnarsson, Rev. Mod. Phys. 61, 689 (1989).

${ }^{27}$ M. Städele, M. Moukara, J. A. Majewski, P. Vogl, and A. Görling, Phys. Rev. B 59, 10031 (1999).

${ }^{28}$ A. D. Becke, J. Chem. Phys. 98, 5648 (1993).

${ }^{29}$ J. Heyd, G. E. Scuseria, and M. Ernzerhof, J. Chem. Phys. 124, 219906 (2006).

${ }^{30}$ L. Hedin, Phys. Rev. 139, A796 (1965).

${ }^{31}$ O. V. Gritsenko, R. van Leeuwen, E. van Lenthe, and E. J. Baerends, Phys. Rev. A 51, 1944 (1995).

${ }^{32}$ A. D. Becke and E. R. Johnson, J. Chem. Phys. 124, 221101 (2006).

${ }^{33}$ N. Umezawa, Phys. Rev. A 74, 032505 (2006).

${ }^{34}$ R. Armiento, S. Kümmel, and T. Körzdörfer, Phys. Rev. B 77, 165106 (2008).

${ }^{35}$ E. Rasanen, S. Pittalis, and C. R. Proetto, J. Chem. Phys. 132, 044112 (2010).

${ }^{36}$ F. Tran and P. Blaha, Phys. Rev. Lett. 102, 226401 (2009).

${ }^{37}$ V. I. Anisimov, F. Aryasetiawan, and A. I. Lichtenstein, J. Phys.: Condens. Matter 9, 767 (1997).

${ }^{38}$ A. Filippetti and V. Fiorentini, Phys. Rev. Lett. 95, 086405 (2005).

${ }^{39}$ D. Wu, Q. Zhang, and M. Tao, Phys. Rev. B 73, 235206 (2006).

${ }^{40}$ S. H. Vosko, L. Wilk, and M. Nusair, Can. J. Phys. 58, 1200 (1980).

${ }^{41}$ E. Engel and R. N. Schmid, Phys. Rev. Lett. 103, 036404 (2009).

${ }^{42}$ A. D. Becke and K. E. Edgecombe, J. Chem. Phys. 92, 5397 (1990).

${ }^{43}$ E. Sim, J. Larkin, K. Burke, and C. W. Bock, J. Chem. Phys. 118, 8140 (2003).

${ }^{44}$ A. Savin, O. Jepsen, J. Flad, O. K. Andersen, H. Preuss, and H. G. von Schnering, Angew. Chem. Int. Ed. Engl. 31, 187 (1992).

${ }^{45}$ B. Silvi and A. Savin, Nature (London) 371, 683 (1994).

${ }^{46}$ A. Savin, A. D. Becke, J. Flad, R. Nesper, H. Preuss, and H. G. von Schnering, Angew. Chem. Int. Ed. Engl. 30, 409 (1991).

${ }^{47}$ M. Nekovee, W. M. C. Foulkes, and R. J. Needs, Phys. Rev. Lett. 87, 036401 (2001).

${ }^{48}$ M. Nekovee, W. M. C. Foulkes, and R. J. Needs, Phys. Rev. B 68, 235108 (2003).

${ }^{49}$ S. J. Clark and P. P. Rushton, J. Phys.: Condens. Matter 16, 4833 (2004).

${ }^{50}$ A. C. Cancio and M. Y. Chou, Phys. Rev. B 74, 081202 (2006).

${ }^{51}$ W. Y. Ching, Y.-N. Xu, and K. W. Wong, Phys. Rev. B 40, 7684 (1989).

${ }^{52}$ K. Terakura, T. Oguchi, A. R. Williams, and J. Kübler, Phys. Rev. B 30, 4734 (1984).

${ }^{53}$ T. C. Leung, C. T. Chan, and B. N. Harmon, Phys. Rev. B 44, 2923 (1991).

${ }^{54}$ P. Dufek, P. Blaha, V. Sliwko, and K. Schwarz, Phys. Rev. B 49, 10170 (1994).

${ }^{55}$ S. Åsbrink and L.-J. Norrby, Acta Crystallogr. Sec. B 26, 8 (1970).

${ }^{56}$ G. Kresse and J. Hafner, Phys. Rev. B 47, 558 (1993).

${ }^{57}$ G. Kresse and J. Hafner, Phys. Rev. B 49, 14251 (1994).

${ }^{58}$ G. Kresse and J. Furthmüller, Phys. Rev. B 54, 11169 (1996).

${ }^{59}$ J. P. Perdew, K. Burke, and M. Ernzerhof, Phys. Rev. Lett. 77, 3865 (1996).

${ }^{60}$ H. J. Monkhorst and J. D. Pack, Phys. Rev. B 13, 5188 (1976). 